DIAS-STP-95-24

October 2018

\title{
Modular Invariance of Finite Size Corrections and a Vortex Critical Phase.
}

\author{
by \\ Charles Nash ${ }^{*}{ }^{\dagger}$ and Denjoe O' Connor ${ }^{\dagger}$ \\ *Department of Mathematical Physics \\ †School of Theoretical Physics \\ St. Patrick's College \\ Dublin Institute for Advanced Studies \\ Maynooth \\ and \\ 10 Burlington Road \\ Ireland \\ Dublin 4 \\ Ireland
}

\begin{abstract}
We analyze a continuous spin Gaussian model on a toroidal triangular lattice with periods $L_{0}$ and $L_{1}$ where the spins carry a representation of the fundamental group of the torus labeled by phases $u_{0}$ and $u_{1}$. We find the exact finite size and lattice corrections, to the partition function $Z$, for arbitrary mass $m$ and phases $u_{i}$. Summing $Z^{-1 / 2}$ over phases gives the corresponding result for the Ising model. The limits $m \rightarrow 0$ and $u_{i} \rightarrow 0$ do not commute. With $m=0$ the model exhibits a vortex critical phase when at least one of the $u_{i}$ is non-zero. In the continuum or scaling limit, for arbitrary $m$, the finite size corrections to $-\ln Z$ are modular invariant and for the critical phase are given by elliptic theta functions. In the cylinder limit $L_{1} \rightarrow \infty$ the "cylinder charge" $c\left(u_{0}, m^{2} L_{0}^{2}\right)$ is a non-monotonic function of $m$ that ranges from $2\left(1+6 u_{0}\left(u_{0}-1\right)\right)$ for $m=0$ to zero for $m \rightarrow \infty$.
\end{abstract}

PACS numbers: 05.40.+j, 05.50.+q, 64.60.-i, 11.15.Ha, 05.70.Fh, 75.10.Hk 
Finite size effects are an intrinsic feature of laboratory experiments which probe the neighbourhood of a continuous phase transition. The scaling properties of these corrections to the infinite (bulk) system behaviour play an increasingly important role in computer simulations and our theoretical understanding of the critical regime of statistical systems [1]. The interplay of these effects with other aspects of the system can give rise to crossover from one characteristic behaviour to another [2]. It is possible to make real progress in the detailed analysis of such situations in two dimensions and for this reason two dimensional models have attracted much interest in recent years. Many of their properties are expressible in terms of those of generalized Gaussian models [3] and with minor modification the results of this note can be adapted to a wide class of other models.

Pure finite size effects can be isolated from those due to the presence of a boundary by ensuring that the connectivity of the underlying lattice is such that it has no boundary. One can avoid other complications, such as local curvature of the lattice, while retaining the finite size effects, by considering a flat torus. Furthermore, in two dimensions, the only zero curvature finite volume manifold without boundary is the flat torus. This torus can be conveniently thought of as a parallelogram with opposite sides identified. We take the sides to be of length $L_{0}$ and $L_{1}$, with $L_{0}$ at an angle $\theta$ to $L_{1}$, cf. fig. 1 . Simple geometry then means that the point $(x, y)$ is thereby identified with the point $\left(x+a L_{1} \cos \theta+b L_{0}, y+\right.$ $\left.a L_{1} \sin \theta\right)$ where $a$ and $b$ are integers.

We consider a triangular lattice composed of similar triangles, pairs of which form parallelograms, cf. fig. 1. The basic triangles have two sides of lengths $a_{0}$ and $a_{1}$ with an angle $\theta$ between them. The complete lattice forms our torus, $T^{2}$, and consists of $K_{0} K_{1}$ sites and $2 K_{0} K_{1}$ triangles, forming a parallelogram of sides $L_{0}=K_{0} a_{0}$ and $L_{1}=K_{1} a_{1}$.

We take a complex continuous spin variable $\varphi(x, y)$ on $T^{2}$ but will not demand that $\varphi$ be periodic, though the underlying lattice is, rather it acquires a phase on being transported 
around one of the cycles of the torus. In general we require that

$$
\varphi\left(x+a L_{1} \cos \theta+b L_{0}, y+a L_{1} \sin \theta\right)=e^{2 \pi i\left(a u_{1}+b u_{0}\right)} \varphi(x, y), \quad a, b \in \mathbf{Z}
$$

For $u_{0}$ and $u_{1}$ neither integer or half integer the spin variable is necessarily either complex or real with two components: From the two component viewpoint these boundary conditions can be thought of as corresponding to vortices winding around the periods of the torus. From a mathematical standpoint $\varphi$ is a section of a bundle $\mathcal{L}$ over the torus $T^{2}$.

Labeling the lattice sites by $\left(k_{0}, k_{1}\right) \equiv \mathbf{k}=k_{0}+\left(k_{1}-1\right) K_{0}$ with $k_{i}=1, \ldots, K_{i}$, the energy of a configuration is given by

$$
\mathcal{E}_{\mathcal{L}}\left[T^{2}, \varphi^{*}, \varphi\right]=\frac{1}{2} \sum_{\mathbf{k k}^{\prime}} \sqrt{g} \varphi^{*}(\mathbf{k})\left(\Delta\left(\mathbf{k}, \mathbf{k}^{\prime}\right)+m^{2} \delta_{\mathbf{k}, \mathbf{k}^{\prime}}\right) \varphi\left(\mathbf{k}^{\prime}\right)
$$

where $\sqrt{g}=a_{0} a_{1} \sin \theta$ and $\delta_{\mathbf{k}, \mathbf{k}^{\prime}}$ is the Kronecker delta. $\Delta$ is a $K_{0} K_{1} \times K_{0} K_{1}$ symmetric matrix, all entries of which are determined by nearest neighbour interactions, so that each spin interacts with six neighbouring spins. Explicitly the only non-zero elements are those given below (and their transposes): we have

$$
\begin{aligned}
\Delta\left\{\left(k_{0}, k_{1}\right),\left(k_{0}+1, k_{1}\right)\right\} & =-\alpha=-\frac{1}{\sin ^{2} \theta}\left(\frac{1}{a_{0}^{2}}-\frac{\cos \theta}{a_{0} a_{1}}\right) \\
\Delta\left\{\left(k_{0}, k_{1}\right),\left(k_{0}, k_{1}+1\right)\right\} & =-\beta=-\frac{1}{\sin ^{2} \theta}\left(\frac{1}{a_{1}^{2}}-\frac{\cos \theta}{a_{0} a_{1}}\right) \\
\Delta\left\{\left(k_{0}+1, k_{1}\right),\left(k_{0}, k_{1}+1\right)\right\} & =-\gamma=-\frac{1}{\sin ^{2} \theta}\left(\frac{\cos \theta}{a_{0} a_{1}}\right) \\
\Delta\left\{\left(k_{0}, k_{1}\right),\left(k_{0}, k_{1}\right)\right\} & =2 \sigma, \quad \text { where } \sigma=\alpha+\beta+\gamma
\end{aligned}
$$

We actually solve the model for general nearest neighbour couplings, $\alpha, \beta$ and $\gamma$, in which case $g=(\alpha \beta+\beta \gamma+\gamma \alpha)^{-1}$. Despite the simplicity of the model we will see that it has a surprisingly rich structure. 
The eigenfunctions of $\Delta$ that satisfy (1) are the discrete Fourier basis

$$
e_{m n}\left(k_{0}, k_{1}\right)=\frac{1}{\sqrt{K_{0} K_{1}}} \exp \left[2 \pi i\left\{\left(m+u_{0}\right) \frac{k_{0}}{K_{0}}+\left(n+u_{1}\right) \frac{k_{1}}{K_{1}}\right\}\right], \quad \text { with }\left\{\begin{array}{l}
m=1, \ldots K_{0} \\
n=1, \ldots K_{1}
\end{array}\right.
$$

orthogonality of which is given by

$$
\sum_{k_{0}=1}^{K_{0}} \sum_{k_{0}=1}^{K_{1}} e_{m n}^{*}\left(k_{0}, k_{1}\right) e_{m^{\prime} n^{\prime}}\left(k_{0}, k_{1}\right)=\delta_{m, m^{\prime}} \delta_{n, n^{\prime}}
$$

while the corresponding sum over $m$ and $n$ gives completeness. The eigenvalues $\lambda_{n_{0} n_{1}}$ of the matrix $\frac{1}{2}\left(\Delta+m^{2}\right)$ are then

$$
\begin{aligned}
\lambda_{n_{0} n_{1}} & =\delta-\alpha \cos \left(x_{n_{0}}\right)-\beta \cos \left(x_{n_{1}}\right)-\gamma \cos \left(x_{n_{0}}-x_{n_{1}}\right) \\
\text { where } x_{n_{i}} & =\frac{2 \pi\left(n_{i}+u_{i}\right)}{K_{i}}, i=0,1 \quad \text { and } \quad \delta=\sigma+\frac{m^{2}}{2}
\end{aligned}
$$

We are interested in the partition function

$$
Z\left(T^{2}, \mathcal{L}, m\right)=\int\left[\prod d \varphi^{*} d \varphi\right] e^{-\frac{1}{k_{B} T} \mathcal{E}_{\mathcal{L}}\left[T^{2}, \varphi^{*}, \varphi\right]}=\prod_{\left(n_{0}, n_{1}\right)}^{\left(K_{0}, K_{1}\right)}\left\{\frac{\pi k_{B} T}{\sqrt{g} \lambda_{n_{0} n_{1}}}\right\}
$$

The free energy is then given by $F=k_{B} T W$ where $W=-\ln Z$.

To perform the sums we note the rearrangement of the eigenvalues

$$
\lambda_{n_{0} n_{1}}=\delta-\alpha \cos x_{n_{0}}-\left|\beta_{n_{0}}\right| \cos \left(x_{n_{1}}-\theta_{n_{0}}\right) \quad \text { where } \quad \beta_{n_{0}}=\beta+\gamma e^{i x_{n_{0}}}=\left|\beta_{n_{0}}\right| e^{i \theta_{n_{0}}}
$$

and use the basic non-trivial identity [4]

$$
\sum_{n=0}^{K-1} \ln \left[z-\cos x_{n}\right]=K \int_{0}^{\frac{\pi}{2}} \frac{d \nu}{\pi} \ln \left[z^{2}-\cos ^{2} \nu\right]+\ln \left|1-\left(z-\sqrt{z^{2}-1}\right)^{K} e^{2 \pi i u}\right|^{2}
$$

where $z \geq 1$ and real. In any case the sum over $n_{1}$ followed by that over $n_{0}$ yields:

$$
W=K_{0} K_{1} W_{B}+W_{F}
$$

where

$$
W_{B}=\int_{-\pi}^{\pi} \frac{d \nu_{1}}{2 \pi} \int_{-\pi}^{\pi} \frac{d \nu_{2}}{2 \pi} \ln \left[\frac{\sqrt{g}}{\pi k_{B} T}\left\{\delta-\alpha \cos \left(\nu_{1}\right)-\beta \cos \left(\nu_{2}\right)-\gamma \cos \left(\nu_{1}-\nu_{2}\right)\right\}\right]
$$


and

$$
\begin{gathered}
W_{F}=K_{1} \sum_{\epsilon= \pm} \int_{0}^{\frac{\pi}{2}} \frac{d \nu}{\pi} \ln \left|1-\left(q_{\epsilon}-\sqrt{q_{\epsilon}^{2}-1}\right)^{K_{0}} e^{2 \pi i u_{0}}\right|^{2} \\
+\sum_{n_{0}=-\left[\frac{K_{0}}{2}\right]}^{\left[\frac{K_{0}-1}{2}\right]} \ln \mid 1-e^{-\left.K_{1} v_{n_{0}} e^{2 \pi i u_{1}}\right|^{2}}
\end{gathered}
$$

here $[x]$ denotes the integer part of $x$. The definitions of the various variables in (12) above are as follows:

$$
q_{ \pm}=q \pm \sqrt{q^{2}-p} \quad q=\frac{\alpha \delta+\beta \gamma \cos ^{2} \nu}{\alpha^{2}}, \quad p=\frac{\delta^{2}-\left(\beta^{2}+\gamma^{2}\right) \cos ^{2} \nu}{\alpha^{2}}
$$

and

$$
v_{n_{0}}=-\ln \left[z_{n_{0}}-\sqrt{z_{n_{0}}^{2}-1}\right]+i \theta_{n_{0}}, \quad \text { where } \quad z_{n_{0}}=\frac{\delta-\alpha \cos x_{n_{0}}}{2\left|\beta_{n_{0}}\right|}
$$

Note that $W_{B}$ gives the free energy per lattice site in the thermodynamic limit, i.e.

$$
\lim _{K_{0}, K_{1} \rightarrow \infty} \frac{W}{K_{0} K_{1}}=W_{B}
$$

hence, due to the fact that we are dealing with a finite rather than an infinite lattice, $W_{F}$ gives the complete finite size correction to the bulk lattice behaviour.

If instead we perform the sum over $n_{0}$ before that over $n_{1}$ we find the alternative composition $W=K_{0} K_{1} W_{B}+\tilde{W}_{F}$. The two apparently different expressions $W_{F}$ and $\tilde{W}_{F}$ are, obviously, equal; they can be transformed into one another by the interchange of $\alpha$ with $\beta$ and the subscripts 0 and 1.

The limit of interest to us is the continuum or scaling limit. This is a constrained thermodynamic limit achieved by taking $K_{0}, K_{1} \rightarrow \infty$ while keeping fixed $L_{i}=K_{i} a_{i}, \theta$, $m^{2}$ and the ratio $k=\frac{K_{1}}{K_{0}}$. The asymptotic form of $W_{B}$ in this scaling limit is given by

$$
W_{B} K_{0} K_{1}=K_{0} K_{1} \Lambda_{B}-\frac{V m^{2}}{4 \pi}\left\{\ln \left[K_{0} K_{1}\right]-2 \rho\right\}-\frac{V m^{2}}{4 \pi}\left(\ln \left[\frac{m^{2} V}{4 \pi^{2}}\right]-1\right)+\cdots
$$


Here $\Lambda_{B}$ is the value of $W_{B}$ at $m=0$ and

$$
\rho \equiv \rho(\alpha, \beta, \gamma)=\int_{0}^{\pi} d \nu\left[\frac{1}{\sin \nu \sqrt{1+g \alpha^{2} \sin ^{2} \nu}}-\frac{1}{\nu}\right]-\frac{1}{2} \ln [\sqrt{g}(\beta+\gamma)]
$$

Despite its appearance, $\rho(\alpha, \beta, \gamma)$ is symmetric under interchange of $\alpha$ and $\beta$. Both $\Lambda_{B}$ and $\rho$ depend on $L_{0}, L_{1}, \theta$ and $k$, or equivalently they depend on the geometry of the lattice triangle.

We find the limiting behaviour of $W_{F}$ is given by $(a)$

$$
\Gamma_{F}=-\frac{\pi \tau_{1}}{6} c\left(u_{0}, \frac{m^{2} V}{\tau_{1}}\right)+\sum_{n=-\infty}^{\infty} \ln \left|1-e^{-2 \pi \tau_{1} \sqrt{\left(n+u_{0}\right)^{2}+\frac{m^{2} V}{4 \pi^{2} \tau_{1}}}+2 \pi i\left\{u_{1}-\tau_{0}\left(n+u_{0}\right)\right\}}\right|^{2}
$$

where

$$
\tau_{0}=\frac{k}{\sqrt{g}|\beta+\gamma|}, \quad \tau_{1}=\frac{k \gamma}{\beta+\gamma} \quad \text { and } \quad V=K_{0} K_{1} \sqrt{g}
$$

The function $c(u, x)$ that appears in (18) is given by

$$
\int_{-\infty}^{\infty} \frac{d p}{2 \pi} \ln \left|1-e^{-\sqrt{p^{2}+x}+2 \pi i u}\right|^{2}=-\frac{c(u, x) \pi}{6}
$$

Corrections to (18) which vanish in the limit are also easily obtainable from (12). In terms of the torus geometry described above we have $\tau_{0}=\frac{L_{1}}{L_{0}} \cos \theta, \tau_{1}=\frac{L_{1}}{L_{0}} \sin \theta$ and $V=L_{0} L_{1} \sin \theta$

Certain special cases of (18) arise in the case of an Ising model on a torus (see [5] where the case $\tau_{0}=0$ was studied). This can be understood from the equivalence of the Ising model to a dimer model on a decorated lattice where similar determinants arise [6]. From this equivalence, exhibiting the phase dependence of $W_{F}$ by $W_{F}\left(u_{0}, u_{1}\right)$, with $\alpha, \beta$, $\gamma$ and $\delta$ obtained from [7], we have

$$
Z^{I \operatorname{sing}}=\frac{1}{2} e^{-W_{B}^{I s i n g}}\left\{\mp e^{\frac{1}{2} W_{F}(0,0)}+e^{\frac{1}{2} W_{F}\left(0, \frac{1}{2}\right)}+e^{\frac{1}{2} W_{F}\left(\frac{1}{2}, 0\right)}+e^{\frac{1}{2} W_{F}\left(\frac{1}{2}, \frac{1}{2}\right)}\right\}
$$

(a) In extracting the limit, the expansions $\ln q_{-}=-\frac{\sqrt{g}}{2}(\beta+\gamma)^{2}\left(\nu^{2}+\frac{g m^{2}}{\beta+\gamma}\right)+\cdots$ and $v_{n_{0}}=\sqrt{\frac{1}{g(\beta+\gamma)|\beta+\gamma|} x_{n_{0}}^{2}+\frac{m^{2}}{|\beta+\gamma|}}+i \frac{\gamma}{\beta+\gamma} x_{n_{0}}+\cdots$ for small $\nu$ and $m^{2}$ are useful. 
for ferromagnetic couplings, with + referring to $T<T_{c}$ and - to $T>T_{c}$. In the scaling limit $W_{F} \rightarrow \Gamma_{F}$. Our results therefore incorporate the complete lattice and finite size corrections for the Ising model on a triangular lattice. With a similar equivalence our results can easily be translated to give the general result for other models.

$\Gamma_{F}$ is invariant under the transformations

$$
\begin{aligned}
& \text { (i) } \quad u_{0} \mapsto u_{0} \quad u_{1} \mapsto u_{0}+u_{1} \quad \tau_{0} \mapsto \tau_{0}+1 \quad \tau_{1} \mapsto \tau_{1} \\
& \text { (ii) } \quad u_{0} \mapsto u_{1} \quad u_{1} \mapsto-u_{0} \quad \tau_{0} \mapsto-\frac{\tau_{0}}{|\tau|^{2}} \quad \tau_{1} \mapsto \frac{\tau_{1}}{|\tau|^{2}}
\end{aligned}
$$

where $\tau=\tau_{0}+i \tau_{1}$. Invariance under (i) is obvious from (18) and records the action of the generator ' $T$ ' of $S L(2, \mathbf{Z}) / \mathbf{Z}_{2}$ while (ii) interchanges $\Gamma_{F}$ with $\tilde{\Gamma}_{F}$ this latter being obtained by performing the sums leading to $\Gamma_{F}$ in the opposite order. These two expressions are equal and (ii) records the action of the generator ' $S$ ' of the modular group $S L(2, \mathbf{Z}) / \mathbf{Z}_{2}$. The volume $V$ is invariant under the action these generators. But $S$ and $T$ generate the whole modular group $S L(2, \mathbf{Z}) / \mathbf{Z}_{2}$ and so $\Gamma_{F}$ and $V$ are invariant under the action of any element of $S L(2, \mathbf{Z}) / \mathbf{Z}_{2}$. Hence we can conclude that in the scaling limit the complete finite size corrections to the bulk free energy are invariant under the entire modular group.

If $M$ denotes an arbitrary element of the modular group, under which $\tau$ undergoes the well known transformation $\tau \mapsto(a \tau+b) /(c \tau+d)$, we have that $\Gamma_{F}$ is invariant under the replacement

$$
\begin{aligned}
& u_{1} \mapsto u_{1}[M]=a u_{1}+b u_{0} \quad u_{0} \mapsto u_{0}[M]=c u_{1}+d u_{0} \\
& L_{1} \mapsto L_{1}[M]=a L_{1}+b L_{0} e^{-i \theta} \quad L_{0} \mapsto L_{0}[M]=c L_{1} e^{i \theta}+d L_{0}
\end{aligned}
$$

where

$$
M=\left(\begin{array}{ll}
a & b \\
c & d
\end{array}\right) \in S L(2, \mathbf{Z}) / \mathbf{Z}_{2}, \quad a, b, c, d \in \mathbf{Z}, a b-c d=1
$$

We note that the sum over the four terms in (21) gives the finite size correction $Z_{F}^{I s i n g}$ as a modular invariant function of $\tau_{0}, \tau_{1}$ and $m^{2} V$. It is clear from our construction that summing over the phases $u_{i}$ which form an orbit of $S L(2, \mathbf{Z}) / \mathbf{Z}_{\mathbf{2}}$ on the space of phases 
(the Picard Variety [8]) will allow one to construct similar phase independent modular invariant partition functions, such as arise in other conformal field theories cf. [9] and references therein.

Taking $m \rightarrow 0$ in (18) we find the critical phase limit of $\Gamma_{F}$ is given by

$$
\begin{aligned}
\Gamma_{F} & =-\frac{\pi \tau_{1}}{6} c\left(u_{0}\right)+\sum_{n=-\infty}^{\infty} \ln \left|1-e^{2 \pi i\left\{\tau|n|-\epsilon_{n}\left(u_{1}-\tau u_{0}\right)\right\}}\right|^{2}, \quad \epsilon_{n}=\frac{n+u_{0}}{\left|n+u_{0}\right|} \\
& =\ln \left|\frac{e^{\pi i u_{0}^{2} \tau} \vartheta_{1}\left(u_{1}-\tau u_{0} \mid \tau\right)}{\eta(\tau)}\right|^{2}
\end{aligned}
$$

where $c(u)=2\{1-6 u(1-u)\}, \vartheta_{1}$ is a Jacobi theta function and $\eta$ is the Dedekind eta function - the modular transformation properties of $\vartheta_{1}$ and $\eta$ are well known and allow an independent check of the modular invariance in this limit.

A further limit of interest is the geometric one obtained by taking $L_{1} \rightarrow \infty$; it corresponds to a cylindrical geometry. In this limit $\Gamma_{F} / V$ reduces to the expression $\gamma^{\text {cylinder }}$ where

$$
\gamma^{\text {cylinder }}=-\frac{\pi}{6 L_{0}^{2}} c\left(u_{0}, m^{2} L_{0}^{2}\right)
$$

(The analogous limit, where $L_{0} \rightarrow \infty$, replaces $u_{0}$ and $L_{0}$ by $u_{1}$ and $L_{1}$ respectively.) When $u_{0}$ and $m$ are both zero the "cylinder charge" $c\left(u_{0}, m^{2} L_{0}^{2}\right)$ reduces to the central charge of the model; in our case $c=2$. The cylinder charge should not be confused with the Zamolodchikov $c$-function [10] for this model, the two functions have different dependencies on $m$, the latter for example being a monotonic function of $m$. We plot $c(u, x)$ in fig. 2 for different values of $u$ as a function of $x$. It exhibits the crossover to $c=0$ at large values of $x$, and is clearly not a monotonic function of $x$. From (21) we see that

$$
\gamma_{\text {Ising }}^{\text {cylinder }}=\frac{\pi}{12 L_{0}^{2}} c\left(\frac{1}{2}, x\right)
$$

Comparison with (25) means that the cylinder charge for the Ising model is $-\frac{1}{2} c\left(\frac{1}{2}, x\right)$, which for $x=0$ gives the usual central charge $c=\frac{1}{2}$ [11]. 
The limits $m \rightarrow 0$ and $u_{0}, u_{1} \rightarrow 0$ for $\Gamma_{F}$ do not commute. To see this we expand $\Gamma_{F}$ (18) for small $m$ and $u_{0}, u_{1}$ obtaining

$$
\Gamma_{F}=\ln \left[(2 \pi)^{2}\left|u_{1}-\tau u_{0}\right|^{2}+\tau_{1} m^{2} V\right]+2 \ln |\eta(\tau)|^{2}+\cdots
$$

and this expression clearly can tend to the distinct logarithmically singular expressions $\ln \left|u_{1}-\tau u_{0}\right|^{2}+2 \ln |\eta(\tau)|^{2}$ and $\ln \left[\tau_{1} m^{2} V\right]+2 \ln |\eta(\tau)|^{2}$ depending on the order in which the limits are taken. However both limits and indeed (27) itself are modular invariant.

The continuum version of the model studied here is described by the Hamiltonian

$$
I\left[\varphi, L_{0}, L_{1}, \theta\right]=\frac{1}{2} \int_{T^{2}} \sqrt{g}\left[\partial_{\mu} \varphi^{*} g^{\mu \nu} \partial_{\nu} \varphi+m^{2} \varphi^{*} \varphi\right]
$$

It is a simple task to obtain the corresponding continuum partition function by the method of $\zeta$-function regularisation. This procedure yields the finite size contribution $\Gamma_{f}$ of (18) but the bulk term differs significantly being now $-\frac{V m^{2}}{4 \pi}\left(\ln \left[(m / 2 \pi \mu)^{2}\right]-1\right)$ where $\mu$ is an arbitrary undetermined scale. Analogues of this continuum model were discussed in [9] where the phases were taken to be rational numbers. Their expressions unfortunately contain errors and they did not succeed in separating the bulk and finite size contributions.

The model we have studied above serves as a useful starting point for a perturbative treatment of the approach to the Kosterlitz-Thouless phase [12] in a $|\varphi|^{4}$ theory and thus captures the neighbourhood of the critical point of the $X Y$ model as the critical point is approached from the disordered phase. That the boundary conditions capture the essential features of a vortex phase can be seen by considering (1) in the cylinder limit and mapping the cylinder to the plane using the conformal map $y+i \frac{2 \pi x}{L_{0}}=\ln z$. The charge of the vortex at the origin is then given by $u_{0}$ and its existence gives rise to an Aharanov-Bohm effect for transport around the origin.

In summary, the finite size corrections to the free energy are modular invariant. This conclusion extends to the entire scaling neighborhood of the critical phase. We use our 
results to give expressions for the complete lattice and finite size corrections for the two dimensional Ising model on a triangular lattice via its equivalence to a sum over Pfaffians. Modular invariance also extends to models in more than two dimensions when the geometry giving rise to finite size effects contains a flat torus. For a three dimensional cylindrical geometry with toroidal cross-section the result can be obtained from $\Gamma_{f}$ by replacing $m^{2}$ with $m^{2}+q^{2}$ and integrating the resulting expression over $q$. One can understand the origin of modular invariance in general as the residual freedom to reparametrize coordinates, in the continuum limit, while retaining flat toroidal geometry.

In the two dimensional case the limiting finite size corrections at the critical phase are expressible in terms of classical elliptic functions. Infinitesimally small values of the phases $u_{i}$ lead to logarithmically divergent contributions to the free energy. This implies that the free energy needed to create a vortex becomes infinite for an infinitely large lattice. In general the model has a surprisingly rich structure of non-commuting limits. For example the limits of approaching the critical phase and that of sending the $u_{i}$ to zero do not commute.

Acknowledgment: We are grateful to Paul Upton for helpful conversations and his careful reading of the manuscript. 
The triangulated torus

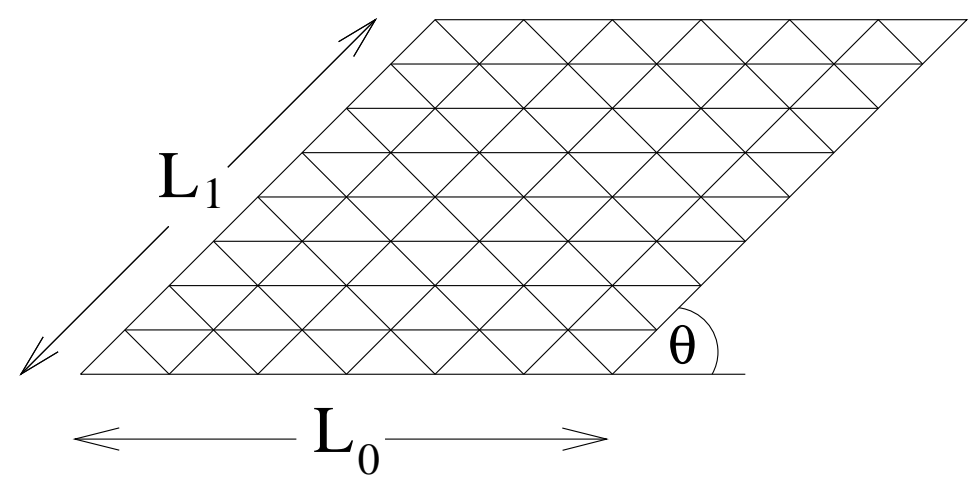

Fig. 1.

The 'cylinder charge' function $c(u, x)$ for various $u$

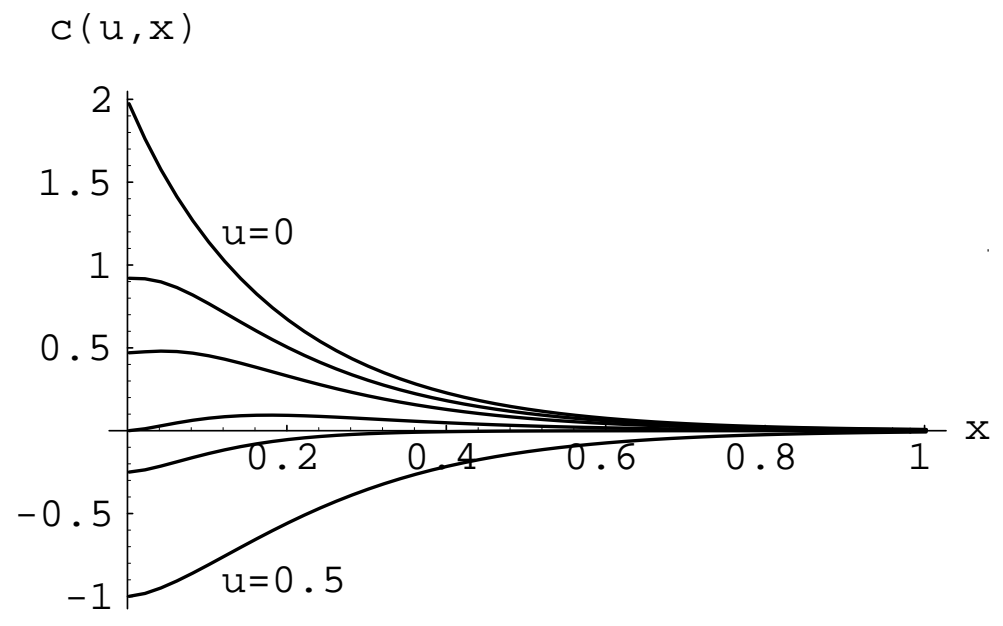

Fig. 2. 


\section{References:}

[1] M. N. Barber, in Phase Transitions and Critical Phenomena, vol.8, eds. C. Domb and J. L. Lebowitz (Academic Press, London 1983). Finite Size Scaling CPSC vol.2, ed. J. L. Cardy (North Holland, 1988).

[2] D. O'Connor and C.R. Stephens, Int. J. Mod. Phys. A9, 2805 (1994).

[3] Vl. S. Dotsenko and V. A. Fateev, Nucl. Phys. B240 312 (1984), and B251, 691 (1985).

[4] This is a generalization of an identity in A. E. Patrick, J. Stat. Phys. 72, 665 (1993).

[5] A. E. Ferdinand and M. E. Fisher, Phys. Rev. 185832 (1969).

[6] P.W. Kastelyn, J. Math. Phys. 4, 287 (1963). M. E. Fisher, J. Math. Phys. 7, 1776 (1966).

[7] J. Stephenson, J. Math. Phys. 8, 1009 (1964).

[10] A. B. Zamolodchikov, Pis'ma Zh. Eksp. Teor. Fiz. 43, 565 (1986) [JETP Lett. 43, $730(1986)$.

[11] H. W. J. Blöte, J. L. Cardy and M. P. Nightingale, Phys. Rev. Lett. 56, 742 (1986). I. Affleck, Phys. Rev. Lett. 56, 746 (1986).

[8] D. B. Ray and I. M. Singer, Ann. Math. 98, 154 (1973).

[9] C. Itzykson and J. Drouffe, Statistical Field Theory vol 2, C.U.P. (1989).

[12] J. M. Kosterlitz and D. J. Thouless, J. Phys. C 6, 1181 (1973). 